\title{
4-Week consumption of anthocyanin-rich blood orange juice does not affect LDL-cholesterol or other biomarkers of CVD risk and glycaemia compared with standard orange juice: a randomised controlled trial
}

\author{
Wendy J. Hollands, Charlotte N. Armah, Joanne F. Doleman, Natalia Perez-Moral, Mark S. Winterbone \\ and Paul A. Kroon* \\ Quadram Institute Bioscience, Norwich Research Park, Norwich NR4 7UA, UK
}

(Submitted 24 August 2017 - Final revision received 17 November 2017 - Accepted 18 December 2017)

\section{Abstract}

Elevated circulating cholesterol levels are a risk factor for CVD which is also associated with sub-optimal vascular function. There is emerging evidence that anthocyanins can cause beneficial cardio-protective effects by favourably modulating lipoprotein profiles. We compared the effects of blood orange juice which is rich in anthocyanins and blonde orange juice without anthocyanins on LDL-cholesterol and other biomarkers of CVD risk, vascular function and glycaemia. In all, forty-one participants (aged 25-84 years) with a waist circumference $>94 \mathrm{~cm}$ (men) and $>80 \mathrm{~cm}$ (women) completed a randomised, open label, two-arm cross-over trial. For $28 \mathrm{~d}$ participants ingested (i) $500 \mathrm{ml}$ blood orange juice providing $50 \mathrm{mg}$ anthocyanins/d and (ii) $500 \mathrm{ml}$ blonde orange juice without anthocyanins. There was a minimum 3-week washout period between treatments. LDL-cholesterol and other biomarkers associated with CVD risk and glycaemia were assessed at the start and end of each treatment period. No significant differences were observed in total, HDL- and LDL-cholesterol, TAG, glucose, fructosamine, nitric oxide, C-reactive protein, aortic systolic blood pressure and diastolic blood pressure or carotid-femoral and brachial-ankle pulse wave velocity after $28 \mathrm{~d}$ ingestion of blood orange juice compared with standard orange juice. The lack of effect on LDL-cholesterol may be due to the modest concentration of anthocyanins in the blood orange juice.

Key words: Human intervention studies: Flavonoids: Polyphenols: Lipids: CVD risk

CVD is a leading cause of mortality worldwide. Atherosclerosis is a widely recognised underlying pathophysiological cause of CVD and is characterised by dyslipidaemia - an elevation in LDL-cholesterol and TAG and a reduction in HDL-cholesterol, either in isolation or in combination. Elevated total cholesterol and LDL-cholesterol are independent risk factors for CVD and interventions (both dietary and pharmaceutical) that aim to reduce cholesterol are central to health strategies to reduce CVD risk in populations.

Anthocyanins are natural plant pigments which confer the red, purple, blue and black colours of anthocyanin-rich foods, such as strawberries, blueberries, blackberries, aubergines and purple potatoes. Anthocyanins comprise an anthocyanidin flavonoid moiety that is O-linked to one or more sugar molecules. In Europe, anthocyanidin intake is reported to range from 3 to $32 \mathrm{mg} / \mathrm{d}$ based on European Food Safety Authority estimates of fruit and vegetable consumption $^{(1)}$ and it is estimated that about $90 \%$ of the total anthocyanin intake is derived from soft fruits. Estimates of anthocyanin intakes in the US range from $4.6 \mathrm{mg} / \mathrm{d}$ (lower quintile) to $19.3 \mathrm{mg} / \mathrm{d}$ (upper quintile) based on reported fruit and vegetable consumption in large cohort studies ${ }^{(2)}$. Although mean intakes of anthocyanins are quite low, single portions of anthocyanin-rich fruits and vegetables can provide very significant quantities of anthocyanins. For example, an $80 \mathrm{~g}$ portion of blackcurrants is reported to contain $574 \mathrm{mg}$ anthocyanins ${ }^{(3)}$.

Epidemiological studies have shown that those consuming the highest quantities of anthocyanins are at a lower risk of developing $\mathrm{CVD}^{(4,5)}$. These observations are substantiated by several human intervention trials providing evidence that ingestion of anthocyanin-rich foods and beverages ${ }^{(6-9)}$, and anthocyanin extracts ${ }^{(10-12)}$ have beneficial effects on markers of CVD risk, with improvements in lipid profiles common across these studies. In a recent systematic review assessing the impact of purified anthocyanins and anthocyanin-rich extracts on biomarkers of CVD risk in both healthy and diseased individuals ${ }^{(13)}$, the authors conclude that supplementation with anthocyanins significantly improved LDL-cholesterol concentrations in hypercholesterolaemic individuals. However, anthocyanin supplementation did not consistently and significantly affect blood pressure, another biomarker of CVD risk.

Blood orange is a variety that is grown mostly in Mediterranean countries such as southern Italy and Spain. It is the

Abbreviations: cf_PWV, carotid-femoral pulse wave velocity; HbA1C, glycated Hb; NO, nitric oxide.

* Corresponding author: Dr P. A. Kroon, email paul.kroon@quadram.ac.uk 
anthocyanins, mainly cyanidin-3-O-glucoside, that is responsible for the red colouration of the flesh of the blood orange. Standard or 'blonde' oranges do not contain anthocyanins. The authors are only aware of a few studies that have investigated the effects of blood orange as a source of anthocyanins on biomarkers for CVD risk. To date, there have been few reports of the effects of blood orange on CVD biomarkers: two reports concerned with effects on platelet function and procoagulant activity $^{(14,15)}$ and one on endothelial function, as assessed by flow mediated dilation of the brachial artery (FMD) ${ }^{(16)}$. However, although blood orange consumption was shown to reduce platelet activity/coagulation ${ }^{(15)}$ and increase flow mediated dilatation $^{(16)}$, neither of these studies could demonstrate that the effects were due to the anthocyanins in blood orange. The aim of this study was to investigate for the first time the effects of daily ingestion of blood orange juice rich in anthocyanins on circulating LDL-cholesterol and other lipids/lipoproteins and on other biomarkers related to vascular function and CVD risk, using blonde orange juice without anthocyanins as a control. In addition, we quantified biomarkers of glycaemia before and after the participants had consumed orange juices for a total of 8 weeks.

\section{Methods}

\section{Study population}

A total of forty-five men and women aged $25-84$ years with a waist measurement $>94 \mathrm{~cm}$ (men) and $>80 \mathrm{~cm}$ (women) were recruited in and around Norwich, UK. The study period was from August 2014 to June 2016. The exclusion criteria were as follows: smoking; medical conditions such as diabetes, heart disease, peripheral vascular disease, gastro-intestinal disease; prescribed and non-prescribed medication judged to affect the study outcome; some dietary supplements (e.g. fish oils); clinical results at eligibility assessment judged to affect the trial outcome or be indicative of a health problem. The trial was conducted in the Human Nutrition Unit at the Quadram Institute Bioscience, Norwich, UK (formerly Institute of Food Research). The study was conducted according to the guidelines laid down in the Declaration of Helsinki and all procedures involving human subjects were approved by both the Human Research Governance Committee of the Quadram Institute Bioscience and the East of England Cambridge and Hertfordshire Research Ethics Committee (reference no. 14/EE/ 0219). Each participant gave written informed consent before taking part in the trial. The trial is registered with clinicaltrials. gov (reference NCT02195934).

\section{Study design}

The study was a randomised, open label, two-arm cross-over trial investigating the effects of $28 \mathrm{~d}$ ingestion of orange juice anthocyanins on biomarkers for CVD. The two treatments were: (i) $500 \mathrm{ml}$ blood orange juice providing $50 \mathrm{mg}$ anthocyanins/d and (ii) $500 \mathrm{ml}$ blonde orange juice without anthocyanins.

Orange juice $(2 \times 250 \mathrm{ml})$ was ingested daily for $28 \mathrm{~d}$ with a minimum 3-week washout period between each of the treatments. Participants were advised to drink the juice with a meal/food.
If this was not possible then, in line with recommendations from the British Dental Association, they were advised to drink the juice through a straw. To aid compliance, participants were provided with a checklist. Each time a drink was consumed, they were instructed to record this on the checklist. Compliance to treatment was assessed from the checklist. For 2 weeks before the start of each treatment period and for the 28-d treatment period, participants completely excluded from the diet some food sources that contribute significantly to total anthocyanin intake (e.g. berry fruits) and limited other food sources known to have cardioprotective effects (e.g. oily fish, dark chocolate, green tea). Limited food sources were completely excluded from the diet for $24 \mathrm{~h}$ before assessment days. Participants were also instructed to completely avoid consuming oranges for the duration of their participation on the trial. A list of prohibited and limited foods was given to the participants to aid compliance.

The primary outcome measure for this trial was LDLcholesterol. Secondary outcome measures were other lipids/ lipoproteins (total- and HDL-cholesterol and TAG), biomarkers of vascular activity and endothelial function (nitric oxide (NO), carotid-femoral pulse wave velocity (cf_PWV); brachialankle pulse wave velocity (ba_PWV); aortic blood pressure), glycaemic control (fasting glucose, fructosamine) and inflammation (high-sensitivity C-reactive protein (CRP)). Primary and secondary outcome measures were assessed before and after the 28-d treatment period. As it was expected that $500 \mathrm{ml}$ of orange juice per $\mathrm{d}$ for a combined total of $56 \mathrm{~d}$ was a substantial 'sugar load', glycated Hb (HBA1c) status was also compared between eligibility assessment and the end of study treatments.

The order in which the participants ingested the two treatments was determined by a computer-generated sequence of letters (randomisation.com). Participants meeting the eligibility criteria were allocated sequentially to a treatment sequence upon enrolment by the study manager.

\section{Orange juice}

Both the standard and blood orange juice were commercially available not from concentrate products sold by Waitrose ('100\% squeezed Sicilian blood orange juice' and 'Tangy and refreshing smooth orange juice') and cartons/bottles were supplied directly by the manufacturer (AMC group). The composition of the juices is shown in Table 1. Juices were

Table 1. Content of orange juice in a 500-ml serving

\begin{tabular}{lcc}
\hline Compounds & Blood orange juice & Blonde orange juice \\
\hline Anthocyanins (mg) & 50 & 0 \\
Hesperidin (mg) & 67 & 104 \\
Narirutin (mg) & 6.5 & 13.5 \\
Energy (kJ) & 1046 & 837 \\
Energy (kcal) & 250 & 200 \\
Fat (g) & 0.5 & 0.5 \\
Carbohydrates (g) & 56.5 & 43.5 \\
of which are sugars (g) & 44.5 & 43.5 \\
Fibre (g) & 2.5 & 4.0 \\
Protein (g) & 3.0 & 3.0 \\
Vitamin C (mg) & 120 & 120 \\
Na (mg) & 0.0 & 0.0 \\
\hline
\end{tabular}


stored frozen $\left(-20^{\circ} \mathrm{C}\right)$ and participants were instructed to defrost the juice in a refrigerator before consumption.

\section{Biochemical markers for CVD}

Whole blood was collected into EDTA, heparin and serum separating tubes (Becton Dickenson) and centrifuged accordingly. Serum lipids (total/HDL-/LDL-cholesterol and TAG), glucose, fructosamine and high-sensitivity CRP status were determined using an automated clinical chemistry analyser (Daytona plus; Randox). Plasma NO metabolites were quantified using a Sievers 280i NO analyser (Sievers Instruments Inc.). The 280i NOA reduces nitrite, nitrate and nitrosothiols to NO in a purge vessel which is then quantified according to the chemiluminescence signal released transiently within the instrument. Plasma samples were deproteinated with cold ethanol (1:2 dilutions) and centrifuged before analysis. Samples $(20 \mu \mathrm{l})$ were injected onto the purge vessel and analysed in triplicate.

\section{Blood pressure assessment}

Measurements were conducted in a quiet room after a 10-min period of rest with the participant in a semi-supine position and the arm resting at heart level. Aortic systolic blood pressure (aSBP) and aortic diastolic blood pressure (aDBP) was measured using a Vicorder device (Smart Medical). Measurements were derived from a peripheral brachial blood pressure measurement and brachial pulse wave analysis using the vicorder in-built transfer function.

\section{Arterial stiffness assessment}

Cf_PWV and ba_PWV were measured with participants in the semi-supine position ( $30^{\circ}$ angle) using a vicorder device. To determine cf_PWV an inflatable sensor $(30 \mathrm{~mm})$ was placed over the right carotid region and a BP cuff placed around the upper right thigh to measure the carotid and femoral pressure pulse waves, respectively. Path length was determined by measuring the distance (in $\mathrm{cm}$ ) between the supra-sternal notch and the mid-point of the thigh cuff. The carotid sensor and thigh cuff were inflated (approximately $60 \mathrm{mmHg}$ ) and waveforms simultaneously recorded over 10-15 consecutive heartbeats. To determine ba_PWV, BP cuffs were placed around the ankle and the right upper arm. Path length was determined by measuring the distance between the supra-sternal notch and the mid-point of the ankle cuff. Both cuffs were inflated and pressure waveforms simultaneously recorded.

\section{Statistical analysis}

The primary outcome measure for this study was LDLcholesterol. To detect a $0.162 \mathrm{mmol} / 1$ decrease in LDLcholesterol at the 5\% significance level and with $80 \%$ power, assuming SD $0.414 \mathrm{mmol} / \mathrm{l}$ in the paired post-intervention differences, forty-two participants were required to complete the study. Multilevel modelling ${ }^{(17)}$ was used to analyse the response variables of interest. The following 'explanatory variables' were the same for all response variables: age, sex, BMI, order of diets given, diet (blonde orange juice and blood orange juice), time (pre- or post-), diet $\times$ time interaction and subject ID (as a random effect). Model diagnostics and best fit criteria were used to decide on the model structure and any necessary transformations and/or outlier removal. The $\mathrm{R}$ statistics package $^{(18)}$ was used to analyse the data. Data were analysed on a per protocol basis. The difference in the concentrations of HbA1c at the end of the study were compared with the concentrations at eligibility assessment using the sign test. All data were considered significant if $P<0 \cdot 05$. All data are presented as means and standard deviations.

\section{Results}

Of the forty-five participants randomised to treatment, forty-one completed the trial (twenty men and twenty-one women). One participant withdrew before commencing the trial because of illness; one participant self-withdrew part way through the first test period citing difficulties with study time commitments as the reason for withdrawal; one was withdrawn by the research team due to difficulties in obtaining blood samples; one developed chest pain and was hospitalised - this was not considered to be related to the trial, but the participant was withdrawn. Fig. 1 shows the flow of participants through the trial and Table 2 shows the baseline demographics and values for biomarkers of CVD at the start of the trial. Compliance to treatments was high with overall compliance of 99.5 and $99.4 \%$; blood orange and blonde orange juice treatments, respectively (juice doses taken as a proportion of intended total) and individual compliance of $\geq 92 \%$.

There were no significant differences at baseline between the treatment groups. After 28-d ingestion of blood orange juice and blonde orange juice, no significant differences between treatments were observed in aSBP, aDBP, circulating lipids (total-/ HDL-/LDL-cholesterol and TAG), cf_PWV, ba_PWV, CRP, NO, glucose and fructosamine (Table 3 ). All values were within reported physiological ranges - the primary outcome measure (LDL-cholesterol) was also assessed with stratification by age ( $<42 ; 42-51 ; 52-61$ and $>61$ years), but no significant difference between treatments was observed.

To assess the effects of juice ingestion on HbA1c status, post study concentrations of HbA1c were compared with the HbA1c concentrations determined at eligibility assessment. No significant difference in HbA1c between the two time points was observed $(P=0 \cdot 3915)$.

\section{Discussion}

We hypothesised that daily ingestion of $500 \mathrm{ml}$ blood orange juice containing anthocyanins for $28 \mathrm{~d}$ would decrease LDLcholesterol compared with a blonde orange juice that did not contain anthocyanins. However, we did not observe a beneficial effect of blood orange juice on either LDL-cholesterol or any of the other biomarkers of vascular function and CVD risk that were measured.

In a recent systematic review conducted by Wallace et al. ${ }^{(13)}$ twelve randomised controlled trials (RCT's) were assessed for the impact of purified anthocyanins and anthocyanin-rich extracts on biomarkers of CVD risk in both healthy and diseased individuals. Nine of the twelve trials included 


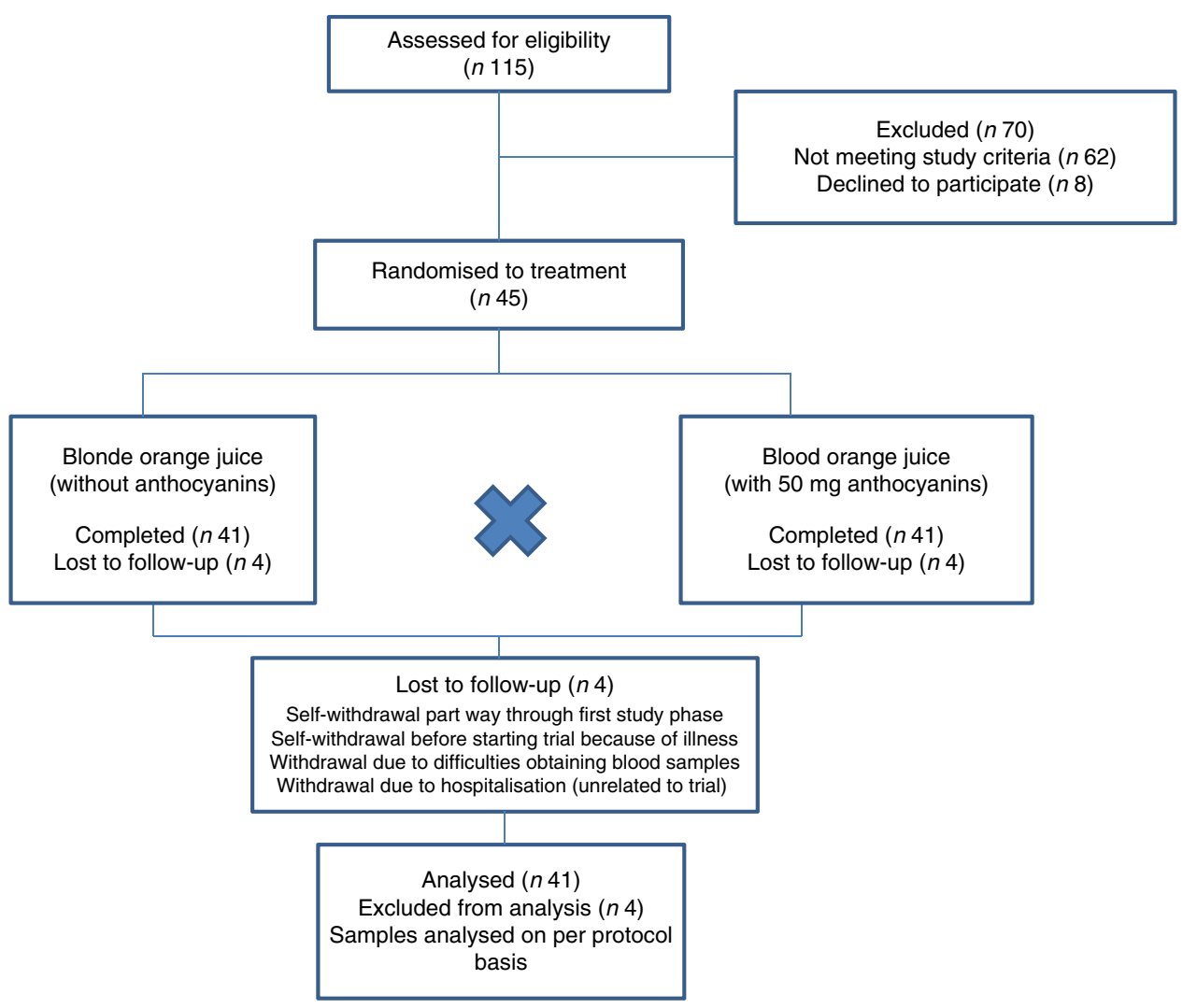

Figure 1. Flow of participants through trial.

Table 2. Baseline demographics and biomarkers of CVD at start of trial (Mean values and standard deviations; $n 41$ )

\begin{tabular}{|c|c|c|c|c|c|c|}
\hline \multirow[b]{2}{*}{ Measurement variables } & \multicolumn{2}{|c|}{ Men $(n$ 20) } & \multicolumn{2}{|c|}{ Women (n 21) } & \multicolumn{2}{|c|}{ Both ( $n$ 41) } \\
\hline & Mean & SD & Mean & SD & Mean & SD \\
\hline Age (years) & $52 \cdot 8$ & $14 \cdot 3$ & 51.6 & $13 \cdot 2$ & $52 \cdot 2$ & $13 \cdot 6$ \\
\hline BMI $\left(\mathrm{kg} / \mathrm{m}^{2}\right)$ & 29.5 & 4.5 & 28.5 & 5.5 & $29 \cdot 0$ & $5 \cdot 1$ \\
\hline Waist measurement $(\mathrm{cm})$ & $105 \cdot 9$ & $11 \cdot 2$ & 91.9 & 11.2 & 98.7 & $13 \cdot 2$ \\
\hline Vicorder aSBP $(\mathrm{mmHg})$ & $126 \cdot 6$ & $9 \cdot 6$ & 123.9 & $12 \cdot 0$ & $125 \cdot 2$ & $10 \cdot 9$ \\
\hline Vicorder aDBP (mmHg) & 74.0 & 6.4 & 68.9 & 8.8 & 71.4 & $8 \cdot 1$ \\
\hline Cf_PWV (m/s) & 7.5 & $1 \cdot 1$ & $7 \cdot 7$ & $1 \cdot 2$ & 7.6 & $1 \cdot 2$ \\
\hline Ba_PWV (m/s) & $13 \cdot 4$ & $2 \cdot 0$ & $12 \cdot 1$ & $2 \cdot 2$ & $12 \cdot 8$ & $2 \cdot 2$ \\
\hline $\mathrm{NO}(\mu \mathrm{m})$ & 34.0 & $12 \cdot 8$ & $33 \cdot 0$ & $10 \cdot 9$ & 33.5 & 11.9 \\
\hline CRP (mmol/l) & $2 \cdot 4$ & $2 \cdot 3$ & 2.5 & $2 \cdot 1$ & 2.4 & $2 \cdot 2$ \\
\hline Glucose (mmol/l) & $5 \cdot 8$ & 0.5 & $5 \cdot 1$ & 0.5 & 5.4 & 0.6 \\
\hline Fructosamine $(\mu \mathrm{mol} / \mathrm{l})$ & 229.5 & 38.2 & $256 \cdot 1$ & $42 \cdot 0$ & $243 \cdot 1$ & $42 \cdot 3$ \\
\hline Total cholesterol $(\mathrm{mmol} / \mathrm{l})$ & $5 \cdot 1$ & 0.9 & $5 \cdot 1$ & 1.0 & $5 \cdot 1$ & 1.0 \\
\hline HDL-cholesterol (mmol/l) & 1.2 & 0.4 & 1.5 & 0.3 & 1.4 & 0.3 \\
\hline LDL-cholesterol (mmol/l) & 3.5 & 0.8 & 3.4 & 1.0 & 3.4 & 0.9 \\
\hline TAG (mmol/l) & 1.4 & 0.7 & 1.3 & 0.8 & $1 \cdot 3$ & 0.8 \\
\hline
\end{tabular}

aSBP, aortic systolic blood pressure; aDBP, aortic diastolic blood pressure; Cf_PWV, carotid-femoral pulse wave velocity; Ba_PWV, brachial-ankle pulse wave velocity; NO, nitric oxide; CRP, C-reactive protein.

LDL-cholesterol as an outcome evaluated for statistical significance in the intervention group compared with the control group. Of these nine trials, five were conducted in healthy individuals or diseased individuals with optimal or near optimal serum lipids and four were conducted in targeted hyperlidaemic individuals. The mean serum total cholesterol and LDL-cholesterol across the five studies of normolipidaemic individuals before intervention was 5.2 and $3.2 \mathrm{mmol} / \mathrm{l}$, respectively. Across the four studies of targeted hyperlidaemic individuals, the mean total cholesterol and LDL-cholesterol at baseline was 6.3 and $3.8 \mathrm{mmol} / 1$, respectively. The authors concluded that anthocyanins significantly improved LDLcholesterol among diseased individuals or those with elevated biomarkers but did not significantly affect other markers of CVD risk. Participants in our trial were recruited with a waist circumference measurement that was indicative of central obesity, which in turn is associated with intra-abdominal fat including visceral adipose tissue (VAT), as there is known to be a positive correlation between VAT and CVD risk factors ${ }^{(19)}$. By association we anticipated that our cohort would present with elevated serum lipid concentrations. The mean total- and LDLcholesterol concentration for the cohort at the start of our trial was 5.1 (SD 1.0) and $3.4(\mathrm{sD} 0.9) \mathrm{mmol} / \mathrm{l}$, respectively, levels in keeping with those values reported in the five trials ${ }^{(20-24)}$ included as part of the systematic review ${ }^{(13)}$ in which no significant reductions in LDL-cholesterol were observed. This may account for the lack of a significant effect of the blood orange juice anthocyanins on LDL-cholesterol in the study reported here.

The differences observed between studies in lipid modulation after anthocyanin ingestion could be ascribed to a variety of factors including length of trial, study population, type of anthocyanin and the daily dose ingested. Two major differences existed between our trial and those reporting significant reductions in LDL-cholesterol that were reported as part of the 
Table 3. Changes in CVD risk markers $28 \mathrm{~d}$ after ingestion of orange juice without anthocyanins (blonde orange juice) and with anthocyanins (blood orange juice) (Mean values and standard deviations)

\begin{tabular}{|c|c|c|c|c|c|c|c|c|c|c|}
\hline \multirow[b]{3}{*}{ Measurement variables } & \multirow[b]{3}{*}{$n$} & \multicolumn{4}{|c|}{ Blonde orange juice } & \multicolumn{4}{|c|}{ Blood orange juice } & \multirow[b]{3}{*}{$P$} \\
\hline & & \multicolumn{2}{|c|}{ Before } & \multicolumn{2}{|c|}{ After } & \multicolumn{2}{|c|}{ Before } & \multicolumn{2}{|c|}{ After } & \\
\hline & & Mean & SD & Mean & SD & Mean & SD & Mean & SD & \\
\hline Total cholesterol $(\mathrm{mmol} / \mathrm{l})$ & 38 & $5 \cdot 1$ & 0.9 & $5 \cdot 2$ & 1.1 & $5 \cdot 1$ & $1 \cdot 1$ & $5 \cdot 1$ & 1.0 & 0.66 \\
\hline LDL-cholesterol (mmol/l) & 38 & 3.4 & 0.9 & 3.6 & 0.9 & 3.5 & 1.0 & 3.6 & 0.9 & 0.87 \\
\hline HDL-cholesterol $(\mathrm{mmol} / \mathrm{l})$ & 38 & 1.4 & 0.3 & 1.3 & 0.4 & 1.3 & 0.4 & 1.3 & 0.3 & 0.72 \\
\hline TAG (mmol/l) & 38 & $1 \cdot 2$ & 0.6 & 1.3 & 0.7 & 1.3 & 0.6 & 1.3 & 0.7 & 0.23 \\
\hline $\mathrm{CRP}(\mathrm{mmol} / \mathrm{l})$ & 38 & $2 \cdot 0$ & 1.8 & 2.6 & 3.5 & 1.9 & 1.4 & $2 \cdot 8$ & 2.9 & $0 \cdot 19$ \\
\hline Nitric oxide $(\mu \mathrm{M})$ & 39 & $35 \cdot 3$ & $12 \cdot 9$ & 37.4 & $15 \cdot 1$ & $35 \cdot 8$ & $12 \cdot 1$ & 36.5 & $13 \cdot 1$ & 0.76 \\
\hline Glucose $(\mathrm{mmol} / \mathrm{l})$ & 38 & 5.7 & 0.7 & 5.4 & 0.6 & $5 \cdot 3$ & 0.7 & $5 \cdot 3$ & 0.7 & 0.20 \\
\hline Fructosamine $(\mu \mathrm{mol} / \mathrm{l})$ & 38 & 241.6 & 43.4 & 249.1 & $42 \cdot 8$ & 244.5 & $42 \cdot 6$ & $244 \cdot 2$ & 45.4 & 0.61 \\
\hline Cf_PWV (m/s) & 41 & $7 \cdot 6$ & 1.2 & $7 \cdot 8$ & 1.7 & $7 \cdot 7$ & $1 \cdot 2$ & 7.9 & $2 \cdot 1$ & 0.31 \\
\hline Ba_PWV (m/s) & 38 & $12 \cdot 7$ & 1.9 & $13 \cdot 0$ & $2 \cdot 2$ & $13 \cdot 1$ & $2 \cdot 1$ & $13 \cdot 0$ & 1.9 & 0.45 \\
\hline $\mathrm{aSBP}(\mathrm{mmHg})$ & 39 & 124 & 11 & 123 & 12 & 124 & 11 & 126 & 14 & 0.87 \\
\hline aDBP $(\mathrm{mmHg})$ & 39 & 71 & 7 & 69 & 8 & 71 & 8 & 69 & 9 & 0.80 \\
\hline
\end{tabular}

CRP, C-reactive protein; Cf_PWV, carotid-femoral pulse wave velocity; Ba_PWV, brachial-ankle pulse wave velocity; aSBP, aortic systolic blood pressure; aDBP, aortic diastolic blood pressure.

systematic review of Wallace et $a l^{(13)}$. First, the daily dose of anthocyanins ingested was higher in three of the four trials ${ }^{(10-12)}$ ranging from 90 to $320 \mathrm{mg}$ anthocyanins/d compared with $50 \mathrm{mg} / \mathrm{d}$ in our own trial. Despite the importance of establishing saturation effects and absorption thresholds after anthocyanin ingestion, few trials report the dose-response effect, which makes it difficult to ascertain the quantity of anthocyanins required to elicit a biological effect such as reductions in circulating cholesterol. After feeding 25 and $50 \mathrm{~g}$ freeze-dried strawberry (FDS)/d providing 78 and $155 \mathrm{mg}$ anthocyanins/d, for a 12-week period to obese individuals with marginally higher than optimal serum lipid concentrations $^{(7)}$ (total and LDL-cholesterol 5.3 and $3.2 \mathrm{mmol} / \mathrm{l}$, respectively), the authors report a significantly greater decrease in LDL-cholesterol after ingestion of the high dose FDS compared with the low dose FDS. However, when compared with the control groups the differences only remained significant at the highest dose of FDS tested. Conversely, in a different trial in which healthy individuals were fed 400 and $1200 \mathrm{mg}$ dried cranberry juice/d, providing 2.6 and $7.8 \mathrm{mg}$ anthocyanins/d, respectively, for a period of 4 and 8 weeks, no significant effect on LDL-cholesterol was observed at either of the two doses or time points tested ${ }^{(25)}$.

In Europe, the mean intake of anthocyanins has been estimated to range between 3 and $32 \mathrm{mg} / \mathrm{d}^{(1)}$, indicating that the dose of anthocyanins ingested by participants in our trial was greater than the average quantities consumed as part of habitual diets. The anthocyanins in blood orange juice are bioavailable in humans as described by Giordano et al. ${ }^{(14)}$. One difference between our trial and those highlighted in the systematic review $^{(13)}$ as causing a reduction in LDL-cholesterol, is the type of anthocyanins ingested. Anthocyanins are present in plants as glycosides and are structurally based on two benzoyl rings, A \& $\mathrm{B}$, coupled with a third heterocyclic $\mathrm{C}$ ring. Anthocyanins are classified according to the number and position of hydroxyl groups at the $\mathrm{B}$ ring, the degree of methylation of the hydroxyl groups and by the number of conjugated sugars attached to the molecule; one or more molecules of glucose, rhamnose, galactose or arabinose to give mono-, di-, or trisaccharide forms. The four trials in the systematic review ${ }^{(13)}$ that reported significant reductions in LDL-cholesterol, were in response to ingestion of anthocyanin extracts of bilberry and blackcurrant $^{(11,12)}$, and whortleberry ${ }^{(10,26)}$ which primarily contain mono- and di-glycosylated delphinidin and malvidin anthocyanins. In contrast, participants in our trial were consuming almost exclusively the cyanidin-3-O-monoglucoside anthocyanin present in blood orange. Moreover, four of the five trials in the systematic review ${ }^{(13)}$ that did not cause a significant reduction in LDL-cholesterol were either in response to ingestion of primarily cyanidin type anthocyanins ${ }^{(20,23)}$ or delphinidin type anthocyanins at doses comparable to those provided in the study reported here ${ }^{(21,22)}$. Anthocyanin absorption is affected by the nature of the sugar moiety which in turn could give rise to differences in its ability to exert a biological effect. A recent animal trial ${ }^{(27)}$ has investigated the metabolic effects of berries containing structurally different anthocyanins in a C57BL/6 mouse model of polygenic obesity. Here, the authors report that animal diets supplemented with blackberry and black raspberry (mono-glycosylated cyanidins), blackcurrant, (mono and diglycosylated cyanidins and delphinidins), maqui berry (diglycosylated delphinidins), concord grape (mono-glycosylated delphinidins and petunidins) and blueberry (monoglycosylated delphinidins, malvidins and petunidins) showed a discrepancy in the biological activities between delphinidin/ malvidin type anthocyanins v. cyanidin type anthocyanins that could be explained by differences in their structure and metabolism in the gut. This strengthens the notion that differences in anthocyanin type could, to some extent at least, explain the discrepancies observed between human intervention trials investigating the effects of anthocyanins on cholesterol.

Anthocyanins have been shown to significantly reduce central blood pressure and cf_PWV, a direct measure of arterial stiffness, in a cross sectional study examining the associations between flavonoid sub-class and biomarkers of CVD risk ${ }^{(28)}$. This is in direct contrast to the results of our trial. However, when a food 
based analysis of the anthocyanin sub-class (e.g. wine and berries) was undertaken, only the reductions in cf_PWV remained significant ${ }^{(28)}$. There are limited published data from trials reporting the effects of anthocyanin-rich foods and beverages on measures of arterial stiffness, and those that do show a significant effect on cf_PWV tend to be from RCT conducted in individuals previously diagnosed with a cardiovascular condition $^{(29)}$.

Although this study was not primarily designed to investigate the possible effects of orange juice consumption on biomarkers of glycaemia, it does provide some useful data. The participants on the study reported here consumed $500 \mathrm{ml}$ of $100 \%$ orange juice for a total of 8 weeks, which provided on average $44 \mathrm{~g}$ of sugars and $941 \mathrm{~kJ} / \mathrm{d}(225 \mathrm{kcal} / \mathrm{d})$ (Table 1$)$. There is a continuing debate about the role of free sugars in causing adverse health effects including obesity, dysregulated metabolic states such as diabetes, CVD and cancer. WHO define free sugars as 'monosaccharides and disaccharides added to foods and beverages by the manufacturer, cook or consumer, and sugars naturally present in honey, syrups, fruit juices and fruit juice concentrates'. The World Health Organization ${ }^{(30)}$ has called on countries to reduce the free sugars intake of adults and children to $<10 \%$ of total daily energy intake although this was based on data from observational studies of the incidence of dental caries. Dietary recommendations in the USA (Dietary Guidelines Advisory Committee ${ }^{(31)}$ ), the UK (Scientific Advisory Committee on Nutrition ${ }^{(32)}$ ) and Canada (Heart and Stroke Foundation Canada ${ }^{(33)}$ ) recommend to reduce energy content from added sugars (= free sugars) to $<10,<5$ and $<10 \%$, respectively. These recommendations were not solely based on prevention of dental caries, but also on reducing energy intakes and evidence of adverse health effects of consuming excess sugars. On the other hand, a systematic review and meta-analysis of prospective cohort studies investigating fruit juice intake and risk of type 2 diabetes showed that higher intakes of sweetened fruit juice was significantly associated with risk of developing type 2 diabetes (relative risk $=1 \cdot 28, P=0 \cdot 02$ ), but no association was observed for higher intakes of $100 \%$ (unsweetened) fruit juice (relative risk $=1.03, P=0.62$ ). In the study reported here, we saw no significant effect of consuming a large dose of sugar-rich $100 \%$ orange juice daily for 8 weeks on circulating HbA1c, and neither did we see any significant increases in fasting glucose or fructosamine after 4 weeks consumption of juice compared with baseline.

One of the main strengths of this study was the cross-over design approach. Participants acting as their own control minimised the influence of confounding variables when assessing the effects of the treatments. In addition, the daily dose of anthocyanins ingested by participants in this study is a dose that is easily achievable as part of a normal diet. This is important from a public health perspective. A potential limitation of this study is that it was conducted in predominantly overweight Caucasian adults and the same results may not be relevant to other population groups such as non-Caucasians and adults of normal weight. In addition, whilst our study population were at higher risk of dyslipidaemia (as determined by abdominal circumference) the mean plasma total cholesterol concentration of the study group was only minimally hyperlidaemic $(5 \cdot 1 \mathrm{mmol} / \mathrm{l})$.
Thus, our data do not preclude the possibility that effects of blood $v$. standard orange juice might occur in participants with more elevated total cholesterol (e.g. $>6.0 \mathrm{mmol} / \mathrm{l}$ ).

In conclusion, our data show that daily ingestion for $28 \mathrm{~d}$ of $50 \mathrm{mg}$ anthocyanins derived from blood orange juice does not reduce LDL-cholesterol or any of the other biomarkers of vascular function and CVD risk measured, and nor did it alter biomarkers of glycaemia. Whether anthocyanin dose or type is important in terms of bioactivity in humans, is yet to be elucidated.

\section{Acknowledgements}

The authors thank Dr Jack Dainty for assistance with statistical analysis of the data, and the AMC group (30100 EspinardoMurcia, Spain) for kindly providing the Sicilian blood orange and standard orange juices that were used in this study.

This research has received funding from the European commission (FP7-ATHENA; grant no. 245121)) and the Biotechnology and Biological Sciences Research Council (UK) through an Institute Strategic Programme Grant ('Food and Health'; grant no: BB/J004545/1) to the Quadram Institute Bioscience (formerly Institute of Food Research). The European Commission and the Biotechnology and Biological Sciences Research Council had no role in the design, analysis or writing of this article.

Author contributions were as follows: P. A. K. designed the research and had primary responsibility for final content; W. J. H., J. F. D., C. N. A., M. S. W. and N. P.-M. conducted the research; W. J. H. and P. A. K. wrote the manuscript. All authors read and approved the final manuscript.

The authors declare that there are no conflicts of interest.

\section{References}

1. Tennant DR, Davidson J \& Day AJ (2014) Phytonutrient intakes in relation to European fruit and vegetable consumption patterns observed in different food surveys. Br J Nutr 112, 1214-1225.

2. Cassidy A, O'Reilly EJ, Kay C, et al. (2011) Habitual intake of flavonoid subclasses and incident hypertension in adults. $\mathrm{Am} \mathrm{J}$ Clin Nutr 93, 338-347.

3. Hollands W, Brett GM, Radreau P, et al. (2008) Processing blackcurrants dramatically reduces the content and does not enhance the urinary yield of anthocyanins in human subjects. Food Chem 108, 869-878.

4. Jennings A, Welch AA, Spector T, et al. (2014) Intakes of anthocyanins and flavones are associated with biomarkers of insulin resistance and inflammation in women. J Nutr $\mathbf{1 4 4}$ 202-208.

5. Wang X, Ouyang YY, Liu J, et al. (2014) Flavonoid intake and risk of CVD: a systematic review and meta-analysis of prospective cohort studies. Br J Nutr 111, 1-11.

6. Alvarez-Suarez JM, Giampieri F, Tulipani S, et al. (2014) Onemonth strawberry-rich anthocyanin supplementation ameliorates cardiovascular risk, oxidative stress markers and platelet activation in humans. J Nutr Biochem 25, 289-294.

7. Basu A, Betts NM, Nguyen A, et al. (2014) Freeze-dried strawberries lower serum cholesterol and lipid peroxidation in adults with abdominal adiposity and elevated serum lipids. J Nutr 144, 830-837. 
8. Murkovic M, Abuja PM, Bergmann AR, et al. (2004) Effects of elderberry juice on fasting and postprandial serum lipids and low-density lipoprotein oxidation in healthy volunteers: a randomized, double-blind, placebo-controlled study. Eur J Clin Nutr 58, 244-249.

9. Erlund I, Koli R, Alfthan G, et al. (2008) Favorable effects of berry consumption on platelet function, blood pressure, and HDL cholesterol. Am J Clin Nutr 87, 323-331.

10. Soltani R, Hakimi M, Asgary S, et al. (2014) Evaluation of the effects of Vaccinium arctostaphylos L. Fruit extract on serum lipids and hs-CRP levels and oxidative stress in adult patients with hyperlipidemia: a randomized, double-blind, placebocontrolled clinical trial. Evid Based Complement Alternat Med 2014, 217451.

11. Qin Y, Xia M, Ma J, et al. (2009) Anthocyanin supplementation improves serum LDL- and HDL-cholesterol concentrations associated with the inhibition of cholesteryl ester transfer protein in dyslipidemic subjects. Am J Clin Nutr 90, 485-492.

12. Zhu Y, Ling W, Guo H, et al. (2013) Anti-inflammatory effect of purified dietary anthocyanin in adults with hypercholesterolemia: a randomized controlled trial. Nutr Metab Cardiovasc Dis 23, 843-849.

13. Wallace TC, Slavin M \& Frankenfeld CL (2016) Systematic review of anthocyanins and markers of cardiovascular disease. Nutrients $\mathbf{8}, 32$.

14. Giordano L, Coletta W, Tamburrelli C, et al. (2012) Four-week ingestion of blood orange juice results in measurable anthocyanin urinary levels but does not affect cellular markers related to cardiovascular risk: a randomized cross-over study in healthy volunteers. Eur J Nutr 51, 541-548.

15. Napoleone E, Cutrone A, Zurlo F, et al. (2013) Both red and blond orange juice intake decreases the procoagulant activity of whole blood in healthy volunteers. Thromb Res 132, 288-292.

16. Buscemi S, Rosafio G, Arcoleo G, et al. (2012) Effects of red orange juice intake on endothelial function and inflammatory markers in adult subjects with increased cardiovascular risk. Am J Clin Nutr 95, 1089-1095.

17. Snijders TAB \& Bosker Roel J (2002) Multilevel Analysis: An Introduction to Basic and Advanced Multilevel Modeling. London: Sage Publications.

18. R Core Team (2015) R: A Language and Environment for Statistical Computing. Vienna: R Foundation for Statistical Computing.

19. Pouliot MC, Despres JP, Lemieux S, et al. (1994) Waist circumference and abdominal sagittal diameter: best simple anthropometric indexes of abdominal visceral adipose tissue accumulation and related cardiovascular risk in men and women. Am J Cardiol 73, 460-468.

20. Curtis PJ, Kroon PA, Hollands WJ, et al. (2009) Cardiovascular disease risk biomarkers and liver and kidney function are not altered in postmenopausal women after ingesting an elderberry extract rich in anthocyanins for 12 weeks. J Nutr 139, 2266-2271.

21. Gurrola-Diaz CM, Garcia-Lopez PM, Sanchez-Enriquez S, et al. (2010) Effects of Hibiscus sabdariffa extract powder and preventive treatment (diet) on the lipid profiles of patients with metabolic syndrome (MeSy). Phytomedicine 17, 500-505.

22. Hansen AS, Marckmann P, Dragsted LO, et al. (2005) Effect of red wine and red grape extract on blood lipids, haemostatic factors, and other risk factors for cardiovascular disease. Eur J Clin Nutr 59, 449-455.

23. Naruszewicz M, Laniewska I, Millo B, et al. (2007) Combination therapy of statin with flavonoids rich extract from chokeberry fruits enhanced reduction in cardiovascular risk markers in patients after myocardial infraction (MI). Atherosclerosis 194, e179-e184.

24. Hassellund SS, Flaa A, Kjeldsen SE, et al. (2013) Effects of anthocyanins on cardiovascular risk factors and inflammation in pre-hypertensive men: a double-blind randomized placebocontrolled crossover study. J Hum Hypertens 27, 100-106.

25. Valentova K, Stejskal D, Bednar P, et al. (2007) Biosafety, antioxidant status, and metabolites in urine after consumption of dried cranberry juice in healthy women: a pilot double-blind placebo-controlled trial. J Agric Food Chem 55 , 3217-3224.

26. Kianbakht S, Abasi B \& Hashem Dabaghian F (2014) Improved lipid profile in hyperlipidemic patients taking Vaccinium arctostaphylos fruit hydroalcoholic extract: a randomized double-blind placebo-controlled clinical trial. Phytother Res 28, 432-436.

27. Overall J, Bonney SA, Wilson M, et al. (2017) Metabolic effects of berries with structurally diverse anthocyanins. Int J Mol Sci 18, 422.

28. Jennings A, Welch AA, Fairweather-Tait SJ, et al. (2012) Higher anthocyanin intake is associated with lower arterial stiffness and central blood pressure in women. Am J Clin Nutr 96, 781-788.

29. Dohadwala MM, Holbrook M, Hamburg NM, et al. (2011) Effects of cranberry juice consumption on vascular function in patients with coronary artery disease. Am J Clin Nutr 93, 934-940.

30. World Health Organization (2015) WHO calls on countries to reduce sugars intake among adults and children. http:// wwwwhoint/mediacentre/news/releases/2015/sugar-guideline/ en/ (accessed August 2017).

31. US Department of Health \& US Department of Agriculture (2015) 2015-2020 Dietary Guidelines for Americans, 8th ed. Washington, DC: US Department of Health \& US Department of Agriculture. https://healthgov/dietaryguidelines/2015/ (accessed August 2017).

32. Scientific Advisory Committee on Nutrition (2015) Carbohydrates and Health report. https://wwwgovuk/government/ publications/sacn-carbohydrates-and-health-report (accessed August 2017).

33. Heart and Stroke Foundation of Canada (2014) Position statement on sugar, heart disease and stroke. https://webarchiveorg/web/ 20160827024321/http://wwwheartandstrokecom/site/cikIQLc MWJtE/b9201361/k47CB/Sugar_heart_disease_and_strokehtm (accessed August 2017). 